\title{
Bolhas Racionais no Índice Bovespa
}

\author{
Mauricio Simiano Nunes*, Sergio Da Silva ${ }^{\dagger}$
}

\author{
Contents: 1. Introdução; 2. Modelo Teórico; 3. Modelos Econométricos; 4. Análise dos \\ Resultados; 5. Conclusão. \\ Keywords: Bolhas Racionais; Bolsas de Valores; Índice Bovespa; Cointegração; Cointegração \\ Threshold. \\ JEL Code: E44; G12.
}

Investigamos a presença de bolhas racionais no comportamento da série do índice Bovespa através de testes de cointegração linear e não linear. Consideramos três tipos de bolha: (1) bolhas explosivas; (2) bolhas que estouram periodicamente e (3) bolhas intrínsecas. Nossos resultados permitem concluir que os tipos (1) e (2) não podem ser descartados na série histórica do índice. Isto significa que, no curto prazo, os preços das ações costumam desviar dos seus fundamentos (pagamentos de dividendos), formando bolhas que acabam em crashes. Isto também significa que as bolhas tendem a ser provocadas por fatores extrínsecos e não pela relação não linear intrínseca entre os preços das ações e os dividendos. We checked for the presence of rational bubbles in the São Paulo Stock Exchange index using both linear and nonlinear cointegration. We detected the presence of explosive and periodically collapsing bubbles, but we could not track intrinsic bubbles.

\section{INTRODUÇÃO}

O valor fundamental de uma ação pode ser definido como a soma de todos os pagamentos esperados de dividendos, levando em conta uma determinada taxa de desconto. Sob a hipótese de eficiência de mercado, os preços das ações variam somente se os investidores reagirem a nova informação relacionada aos fundamentos. Isto significa que os preços seguem um martingale e que qualquer desvio sistemático em relação ao valor fundamental deve ser considerado uma bolha.

Nesse contexto de bolhas racionais, podemos destacar três tipos específicos:

(1) bolhas explosivas, que ocorrem na ausência de equilíbrio de longo prazo entre o preço da ação e o pagamento de dividendos;

(2) bolhas que estouram periodicamente, que são aquelas que, ultrapassado certo valor limite, decaem até um valor próximo de zero, mas voltam a crescer lentamente até o alinhamento de longo prazo entre o preço da ação e os dividendos; e

\footnotetext{
* Programa de Pós-Graduação em Economia, Universidade Federal do Rio Grande do Sul. E-mail: mauriciosimianonunes@ gmail.com

†Programa de Pós-Graduação em Economia, Universidade Federal de Santa Catarina. E-mail: professorsergiodasilva@ gmail.com
} 
(3) bolha intrínsecas, que, ao contrário das anteriores, surgem meramente da reação excessiva do preço da ação em relação à variação dos dividendos, não havendo, portanto, atuação de fatores extrínsecos.

Bolhas nos preços dos ativos podem ser relevantes em economias emergentes principalmente por causa do grande volume de capitais recebidos por esses países nas últimas duas décadas. Como estes influxos podem acarretar efeitos colaterais (Mundial (1997), Sarno and Taylor (1999a,b)) e potencial reversibilidade, as autoridades de política precisam se precaver ao utilizarem capitais externos visando objetivos econômicos domésticos. Os efeitos colaterais incluem as distorções provocadas no processo de determinação dos preços de ativos e nas taxas de câmbio reais. Também incluem as bolhas nos preços de ativos que são seguidas de estouro. Isto torna o ciclo econômico desnecessariamente mais volátil (Helbling and Terrones, 2003). Além disso, uma bolha nos preços das ações pode gerar redução do prêmio pelo financiamento externo não devidamente fundamentado, acarretando excessivo investimento em capital e euforia seguida de crash e recessão. Com falta de informação, também pode ocorrer comportamento de "manada", onde um grande número de indivíduos reage da mesma maneira diante de nova informação; isto pode provocar uma reação excessiva em termos agregados (Bikhchandani and Sharma, 2001). Bancos com insuficiente informação a respeito das intenções de investimento dos seus devedores, por exemplo, podem subestimar o risco na concessão de empréstimos e exagerar no crédito, o que pode provocar a formação de uma bolha. Muitas ocorrências de crises econômicas ou financeiras estiveram historicamente associadas à formação de bolhas nos preços dos ativos, como bem demonstra a recente bolha de propriedade.

Pode-se estudar a presença de bolhas nos preços de ações de maneira teórica (como em Hamilton and Whiteman (1985), Campbell and Shiller (1988) e Santos and Woodford (1997)) ou econométrica. Alguns estudos econométricos procuram detectar bolhas nos preços das ações através de mudanças de regime markoviano. Hall and Sola (1993) fizeram um teste com probabilidade de mudança de regime constante e encontraram bolhas no índice S\&P 500. Mas se a probabilidade de mudança de regime não for constante e for função do tamanho da bolha, esta desaparece (Van Norden (1996), Van Norden and Vigfusson (1998)). Driffill and Sola (1998) também procuraram, sem sucesso, detectar bolhas intrínsecas através do modelo de mudança de regime para os dividendos. Para o Brasil, Martin et al. (2004) encontraram bolhas no índice Bovespa utilizando a mesma metodologia de mudança de regime markoviana.

Taylor and Peel (1998) propõem um teste alternativo via cointegração robusta a assimetria e curtose nos erros. Com simulações de Monte Carlo, eles confirmam que seu modelo é superior a um teste simples via Dickey-Fuller para detectar bolha. Embora eles não tenham encontrado evidência de bolha nos dados da economia americana entre 1887 e 1987, há evidência, usando-se a mesma metodologia, para alguns países da América Latina, incluindo o Brasil (Sarno and Taylor (2003) e Herrera and Perry (2003)).

Bidarkota and Dupoyet (2007) sugerem um teste para bolhas intrínsecas considerando uma distribuição de Lévy estável (em vez da Gaussiana) para os resíduos da equação dos dividendos. Caudas grossas levam a uma menor discrepância entre os preços observados e os preços compatíveis com os fundamentos (dados por um modelo de valor presente, por exemplo) e o papel do componente de bolha intrínseca nas variações observadas dos preços das ações se reduz. Contudo, Ma and Kanas (2004) testam um modelo de cointegração não linear entre os preços das ações e os dividendos e encontram forte evidência em favor da hipótese de bolhas intrínsecas para os Estados Unidos.

Bohl and Siklos (2004), por sua vez, testam a hipótese de bolhas que estouram periodicamente e encontram que, no curto prazo, os preços do mercado de ações americano apresentam grandes e persistentes bolhas seguidas de estouro. No longo prazo, porém, há suporte empírico para o modelo de valor presente (a ser apresentado em detalhes na Seção 2). Payne and Waters (2005) também encontram evidência de ajustamento assimétrico em direção ao equilíbrio de longo prazo entre os preços no mercado imobiliário americano e os fundamentos, com os preços ficando, muitas vezes, abaixo dos valores justificados pelos fundamentos, indicando a presença de bolhas negativas. 
O preço fundamental pode ser medido de outras maneiras. Por exemplo, no modelo do Fed leva-se em conta a comparação dos rendimentos dos salários com os dos títulos e a forte correlação existente entre salários, o índice S\&P 500 e os rendimentos do Tesouro. No modelo do Banco do Canadá considerase a tendência estocástica comum entre os preços das ações e variáveis macroeconômicas (Gauthier and Li, 2004). Esta abordagem foi adotada no trabalho mencionado de Herrera and Perry (2003) e também pode ser útil para o Brasil, já que há evidência de relação entre os preços dos ativos e as variáveis macroeconômicas (Nunes et al., 2005).

Assim, neste artigo investigaremos a presença de bolhas racionais no índice Bovespa através de testes de cointegração linear e não linear. Levaremos em conta os três tipos de bolha acima mencionados. Os resultados vão mostrar a presença de bolhas explosivas e de bolhas que colapsam periodicamente na série histórica do índice. Os resultados também irão sugerir que essas bolhas devem ter sido provocadas por eventos extrínsecos. Ou seja, não foram bolhas intrínsecas advindas da relação não linear entre os preços das ações e os pagamentos de dividendos.

Na Seção 2 apresentaremos o modelo teórico de bolha racional. Na Seção 3 explicaremos os testes econométricos para se estimar bolhas explosivas, bolhas que estouram periodicamente e bolhas intrínsecas. Na Seção 4 descreveremos os dados e faremos a análise dos nossos resultados. As conclusões serão expostas na Seção 5.

\section{MODELO TEÓRICO}

O modelo pioneiro de bolha racional foi o de Blanchard (1979) (veja também Blanchard and Watson (1982)). No modelo, as bolhas são provocadas por expectativas “auto-realizáveis". Agentes com expectativas de preço racionais não cometem erros sistemáticos e, portanto, a relação positiva entre o preço corrente e sua variação futura esperada implica relação igualmente positiva entre o preço corrente e sua variação observada. É neste sentido que as expectativas se "auto-realizam": direcionando o preço corrente no sentido das expectativas, de maneira independente dos fundamentos. Qualquer desalinhamento pode ser explicado por reações racionais a restrições existentes no mundo real.

Nesse ambiente, o apreçamento das ações é feito a partir da maximização da utilidade

$$
\max E_{t}\left(\sum_{i=0}^{\infty} \beta^{i} u\left(c_{t+i}\right)\right)
$$

s.a.

$$
c_{t+i}=y_{t+i}+\left(P_{t+i}+D_{t+i}\right) x_{t+i}-P_{t+i} x_{t+i+1}
$$

Aqui, $y_{t}$ é a dotação inicial; $\beta$ é a taxa de desconto do consumo futuro; $x_{t}$ é o estoque de ações; $P_{t}$ é o preço da ação depois da distribuição dos dividendos; e $D_{t}$ é o próprio dividendo. A condição de primeira ordem deste problema é

$$
E_{t}\left(\beta u^{\prime}\left(c_{t+i}\right)\left(P_{t+i}+D_{t+i}\right)\right)=E_{t}\left(u^{\prime}\left(c_{t+i+1}\right) P_{t+i-1}\right)
$$

Se a utilidade for linear e, portanto, a utilidade marginal for constante, e o consumidor for neutro ao risco, a equação (3) torna-se

$$
\beta E_{t}\left(P_{t+i}+D_{t+i}\right)=E_{t}\left(P_{t+i-1}\right)
$$

Supondo a existência de um ativo sem risco e a taxa de juros aplicável para apenas um período, a condição de não arbitragem implica

$$
E_{t}\left(P_{t+i-1}\right)=\frac{1}{1+r} E_{t}\left(P_{t+i}+D_{t+i}\right)
$$


onde $r$ é a taxa de juros para um período. Esta equação em diferenças de primeiro grau é o ponto de partida para a maioria dos modelos de apreçamento de ações. Iterando-a para frente encontramos a solução

$$
P_{t}=\sum_{i=1}^{\infty}\left(\frac{1}{1+r}\right)^{i} E_{t}\left(D_{t+i}\right)+B_{t}
$$

tal que

$$
E_{t}\left(B_{t+i}\right)=(1+r) B_{t}
$$

Portanto, o preço da ação possui dois componentes:

(1) o relativo aos fundamentos do mercado, que é o valor descontado dos dividendos futuros esperados (primeiro termo à direita da igualdade na equação (6)); e

(2) o relativo à bolha, $B_{t}$ (segundo termo).

A bolha é racional porque não se deve a nenhum erro de apreçamento: ela é simplesmente um dos componentes do preço da ação. Apesar da possibilidade de bolha, a equação 7 mostra que não há oportunidade de arbitragem. Se os dividendos crescerem mais lentamente do que $r$, o componente relativo aos fundamentos irá convergir. Porém, o componente da bolha é não estacionário. Se os agentes acreditarem que podem vender a ação por um preço mais alto em data futura, o preço da ação pode exceder o valor fundamental.

A equação 7 impõe uma restrição ao movimento da bolha, mas é compatível com uma trajetória diferente para cada valor inicial possível dela. A trajetória do preço da ação não é única. Para que seja, é necessária uma suposição adicional em relação a $B_{t}$. Uma possível suposição é fixar $B_{t}=0$ em todos os períodos. Como o preço corrente da ação é a soma dos valores presentes líquidos dos dividendos esperados e do valor de revenda esperado, i.e.

$$
P_{t}=\sum_{i=1}^{\infty}\left(\frac{1}{1+r}\right)^{i} E_{t}\left(D_{t+i}\right)+\lim _{i=\infty}\left(\frac{1}{1+r}\right)^{i} P_{t+i}
$$

impõe-se a condição de transversalidade para zerar o segundo termo à direita da igualdade na equação 8 . A condição de transversalidade é justificada pelo argumento de que, havendo bolha, o agente com vida infinita pode, cedo ou tarde, vender a ação. A perda de utilidade advinda do valor descontado do fluxo dos dividendos será menor do que o valor da venda. Este preço de venda não pode ser de equilíbrio porque todos os agentes venderão a ação fazendo com que o preço caia até o valor fundamental.

Em resumo, a bolha é racional por não se dever a qualquer erro de apreçamento; antes, ela é um dos componentes do preço da ação. O modelo de valor presente, comumente utilizado em finanças, deriva da imposição da condição de transversalidade que visa zerar o componente referente à bolha.

\section{MODELOS ECONOMÉTRICOS}

Os modelos econométricos procuram verificar a presença ou não das bolhas racionais acima em três situações distintas: de bolhas explosivas, de bolhas periódicas e de bolhas intrínsecas.

\subsection{Bolhas explosivas}

Na solução geral do modelo de apreçamento das ações (equação 8) não há oportunidade para arbitragem (equação 7). Como os preços não podem ser negativos, também não pode haver retorno excessivo (Diba and Grossman, 1987, 1988b). Isto significa que a bolha $B_{t}$ deverá ser a solução da equação em diferenças expectacional

$$
B_{t+1}-(1+r) B_{t}=0
$$


Qualquer valor diferente de zero para $B_{t}$ comprovará a existência de bolha racional. A solução da equação 9 segue uma equação em diferença estocástica do tipo

$$
B_{t+1}-(1+r) B_{t}=z_{t+1}
$$

onde $z_{t+1}$ é uma variável aleatória (ou uma combinação de variáveis) gerada por um processo estocástico que satisfaz

$$
E_{t-j}\left(z_{t+1}\right)=0
$$

para qualquer $j \geq 0$. Para $B_{t}$ igual a zero, todas as realizações futuras de $z$ devem ser zero com probabilidade 1 e, assim, a bolha não pode se iniciar. Portanto, se há bolha, ela precisa ter existido desde o primeiro dia da transação (Diba and Grossman, 1987, 1988b).

Mesmo que o preço fundamental não seja observado, o teste sugerido por Diba and Grossman (1988a) mostra que os desvios dos fundamentos não observados podem, eles mesmos, ser observados, caso exista a bolha. $O$ preço fundamental de mercado $P_{t}^{f}$ será dado por

$$
P_{t}^{f}=\sum_{i=1}^{\infty}\left(\frac{1}{1+r}\right)^{i} E_{t}\left(D_{t+i}+\sigma_{t}\right)
$$

onde $D_{t+i}$ representa os dividendos futuros esperados e $\sigma_{t}$ é o fundamento não observado. Na ausência de bolha, se os dividendos forem estacionários em nível, o preço da ação será igual ao valor fundamental e, portanto, também deverá ser estacionário em nível. Se os dividendos forem estacionários na enésima diferença, o preço da ação deverá ser estacionário na enésima diferença. Existindo bolha (e considerando a equação (10)) a enésima diferença será dada por

$$
(1-L)^{n}\left(1-(1+r) B_{t}\right)=(1-L)^{n} z_{t}
$$

onde $L$ é o operador de defasagens. Se $z_{t}$ for um ruído branco, a primeira diferença será gerada por um processo não estacionário e não inversível. A trajetória da bolha será não estacionária, não importando quantas diferenças sejam consideradas. Portanto, a existência de bolha pode ser testada verificando se o preço da ação permanece estacionário depois de tomadas as diferenças o número suficiente de vezes para tornar os dividendos estacionários.

A equação 12 impõe uma relação de equilíbrio entre as duas variáveis. Supondo que $\sigma_{t}$ seja estacionário, sob a hipótese nula de ausência de bolha, o preço da ação e os dividendos precisam ser cointegrados. Isto significa que $P_{t}-\frac{1}{r} D_{t}$ é estacionário na ausência de bolha explosiva.

Diba and Grossman (1988a) encontraram que os preços das ações de empresas norte-americanas e os seus dividendos são integrados em níveis, mas estacionários em primeiras diferenças. As séries são, portanto, cointegradas, indicando a ausência de bolhas explosivas e, portanto, a validade no longo prazo do modelo de valor presente.

\subsection{Bolhas que estouram periodicamente}

Evans (1991) discorda de que a impossibilidade de rejeitar a hipótese de estacionaridade-cointegração seja prova da não existência de bolha. Para ele é possível que a bolha estoure para um valor pequeno, mas diferente de zero, depois continuando a crescer sem violar a equação 7. Estas "bolhas que estouram periodicamente" são descritas como

$$
\begin{array}{lr}
B_{t+1}=(1+r) B_{t} u_{t+1} & \text { se } B_{t} \leq \tau \\
B_{t+1}=\left(\delta+\pi^{-1}(1+r) \theta_{t+1}\left(B_{t}-(1+r)^{-1} \delta\right)\right) u_{t+1} & \text { se } B_{t}>\tau
\end{array}
$$


onde $\delta$ e $\alpha$ são parâmetros positivos com $0<\delta<(1+r) \tau$; $u_{t+1}$ é uma variável aleatória exógena, positiva, independente e identicamente distribuída (i.i.d.), e com $E_{t}\left(u_{t+1}\right)=1 ; \theta_{t+1}$ é uma variável exógena i.i.d., que segue um processo de Bernoulli, e que apresenta o valor de 1 com probabilidade $\mu$, e de 0 com probabilidade $1-\mu$. O processo (14) satisfaz (7) e, para valores pequenos de $B_{t}$, a bolha cresce lentamente à taxa média $1+r$. Ultrapassado o valor limite $\tau$, a bolha se expande rapidamente, podendo estourar com probabilidade $1+\pi$. Depois do estouro, o valor da bolha não retorna a zero, mas a um pequeno valor médio $\delta$, quando então o processo se reinicia.

A presença de bolhas que estouram periodicamente pode ser detectada por cointegração threshold auto-regressiva (TAR) (Tong, 1983) e por sua variante: momentum threshold autoregressive cointegration (M-TAR) (Enders and Granger (1998), Enders and Siklos (2001)). Estes modelos não lineares são mais poderosos do que os modelos convencionais de cointegração (Engle and Granger (1987), Johansen (1988, 1991)), pois capturam ajustamentos assimétricos.

Os modelos de raiz unitária e cointegração convencionais podem não ser aqui apropriados porque supõem uma raiz unitária como hipótese nula e um processo linear como hipótese alternativa, além de considerarem que o ajustamento é simétrico. Já a cointegração TAR permite um grau de queda autoregressiva que depende do estado da variável em estudo. O modelo M-TAR vai além, ao permitir que a variável apresente diferentes valores de decaimento auto-regressivo: crescente ou decrescente. Assim, ele pode capturar a possibilidade de assimetria no ajustamento de curto prazo dos preços das ações e, desta forma, pode testar tanto o modelo de valor presente (bolhas explosivas) como o das bolhas que estouram periodicamente (descritas na equação 14). Enders and Granger (1998) e Enders and Siklos (2001) fizeram testes com séries aleatórias para avaliar o poder de teste relativo dos modelos TAR e M-TAR comparando-os com o modelo Engle-Granger padrão. Eles encontraram que o modelo M-TAR apresenta melhor desempenho do que o modelo TAR.

Assim, a especificação TAR/M-TAR para se detectar bolha no preço de ações parte da relação de cointegração entre preço da ação $P_{t}$ e os dividendos $D_{t}$, i.e.

$$
P_{t}=\hat{\beta}_{0}+\hat{\beta}_{1} D_{t}+\hat{\mu}_{t}
$$

onde $\hat{\beta}_{0}$ e $\hat{\beta}_{1}$ são os parâmetros da equação de regressão. Se há bolhas que estouram periodicamente, o resíduo estimado $\hat{\mu}_{t}$ da regressão de cointegração (15) irá capturar a seqüência de aumentos de preço da ação seguida de queda repentina. Este comportamento particular pode ser expresso como

$$
\triangle \hat{\mu}_{t}=I_{t} \rho_{1} \hat{\mu}_{t-1}+\left(1-I_{t}\right) \rho_{2} \hat{\mu}_{t-1}+\sum_{i=1}^{l} \gamma_{i} \Delta \hat{\mu}_{t-1}+\varepsilon_{t}
$$

onde $I_{t}$ é uma função indicadora definida como

$$
I_{t}=\left\{\begin{array}{lll}
1 & \text { se } & \hat{\mu}_{t-1} \geq \tau \\
0 & \text { se } & \hat{\mu}_{t-1}<\tau
\end{array}\right.
$$

sendo $\tau$ o valor do threshold. O modelo TAR considera como hipótese nula a não existência de cointegração, i.e. $H_{0}: \rho_{1}=0, H_{0}: \rho_{2}=0$ e $H_{0}: \rho_{1}=\rho_{2}=0$. Aqui, Enders and Siklos (2001), Tabelas 1 e 2 provêem os valores críticos para os testes $t$ e $F$ correspondentes. Se a hipótese nula de não cointegração for rejeitada, a hipótese de ajustamento simétrico $H_{0}: \rho_{1}=\rho_{2}$ deve ser testada utilizando-se a estatística $F$. No caso de a hipótese nula $H_{0}: \rho_{1}=\rho_{2}=0$ não poder ser rejeitada, pode-se concluir que a relação de cointegração entre $P_{t}$ e $D_{t}$ apresenta ajustamento linear e simétrico.

Petruccelli and Woolford (1984) mostraram que as condições necessárias e suficientes para a estacionaridade de $\left\{\hat{\mu}_{t}\right\}$ são $\rho_{1}, \rho_{2}<0$ e $\left(1+\rho_{1}\right)\left(1+\rho_{2}\right)<1$ para qualquer valor de $\tau$. Se a série convergir, $\hat{\mu}=0$ será o valor de equilíbrio de longo prazo da seqüência. Se $\hat{\mu}_{t-1}$ ficar abaixo do equilíbrio de longo prazo, o ajustamento será dado por $\rho_{2} \hat{\mu}_{t-1}$. Como o ajustamento será simétrico quando $\rho_{1}=\rho_{2}, \mathrm{o}$ 
modelo de cointegração de Engle and Granger (1987) passa a ser um caso especial do modelo TAR. Um exemplo de como o modelo TAR pode capturar movimentos abruptos em uma seqüência seria: se $-1<\rho_{1}<\rho_{2}<0$, então a fase negativa da seqüência $\left\{\hat{\mu}_{t}\right\}$ tenderá a ser mais persistente do que a fase positiva (Enders and Granger, 1998). Portanto, a presença de bolhas que estouram periodicamente pode ser capturada através da acumulação das variações de $\hat{\mu}_{t-1}$ acima do threshold, seguida de queda abrupta no threshold. Mas a trajetória das variações de $\hat{\mu}_{t-1}$ abaixo do threshold não mostra sinais de erupções de bolhas seguidas de colapso.

Como exemplo podemos supor que $\tau=0$ em (16). Um valor $\hat{\mu}_{t-1}>0$ será então indicativo de aumento no preço da ação em relação aos dividendos, seguido de quebra. Já uma acumulação de reduções no preço da ação em relação aos dividendos $\hat{\mu}_{t-1}<0$, seguida de súbito aumento de volta ao caminho de equilíbrio, não seria esperada. Isto mostra assimetria nos desvios do equilíbrio e indica a existência de uma bolha que estoura periodicamente. Se, além disso, o coeficiente estimado $\hat{\rho}_{1}$ for estatisticamente significativo, negativo e maior em termos absolutos do que o parâmetro $\hat{\rho}_{2}$, a hipótese nula de ajustamento simétrico $H_{0}: \rho_{1}=\rho_{2}$ será rejeitada (Bohl, 2003).

As hipóteses nulas do modelo convencional de Engle-Granger e as do modelo TAR são idênticas, mas suas hipóteses alternativas diferem no caso da rejeição da hipótese nula $H_{0}: \rho_{1}=\rho_{2}$. Testar a hipótese nula de ausência de cointegração (contra a alternativa de cointegração com ajustamentos TAR) permite a detecção de bolhas que estouram periodicamente. Mesmo que isto seja feito de modo indireto no modelo TAR, esta desvantagem é menor do que aquelas presentes nos testes de raiz unitária e nos modelos de cointegração convencionais.

No modelo TAR, a função indicadora considera os níveis dos resíduos da equação de cointegração $\hat{\mu}_{t-1}$, como vimos. Alternativamente, Enders and Granger (1998) e Caner and Hansen (2001) sugerem que o threshold dependa das variações dos resíduos do período anterior $\left\{\triangle \hat{\mu}_{t-1}\right\}$. Neste caso, a função indicadora (17) deve ser substituída por

$$
I_{t}=\left\{\begin{array}{lll}
1 & \text { se } & \Delta \hat{\mu}_{t-1} \geq \tau \\
0 & \text { se } & \Delta \hat{\mu}_{t-1}<\tau
\end{array}\right.
$$

As equações 15, 16 e 18 constituem o M-TAR: agora a série exibe mais "ímpeto" (momentum) em uma direção do que na outra (Enders and Siklos, 2001).

Enders and Granger (1998) e Enders and Siklos (2001) calcularam os valores críticos dos modelos TAR e M-TAR através de simulações de Monte Carlo e bootstrap. A estatística $t$ para a hipótese nula $\rho_{1}=0$ e $\rho_{2}=0$ é reportada juntamente com a estatística $F$ para a hipótese nula $\rho_{1}=\rho_{2}=0$. A mais significativa das estatísticas $t$ foi chamada de $t_{\max }$; a menos significativa é a $t_{\min }$, e a estatística $F$ passou a ser a $\phi$, que apresenta maior poder. Por isso, na condição de convergência $\rho_{1}<0$ e $\rho_{2}<0$, é a estatística $\phi$ que rejeita a hipótese nula de ausência de cointegração. A estatística $\phi$ somente deve ser utilizada se as estimativas no ponto de $\rho_{1}$ e $\rho_{2}$ implicarem convergência. Porém, se algum $\rho$ for positivo, deve-se recorrer à estatística $t_{\max }$.

Em princípio, o threshold não precisa coincidir com o atrator zero, e $\tau$ pode ser estimado em conjunto com $\rho_{1}$ e $\rho_{2}$. Chan (1993) propõe encontrar $\tau$ ordenando de forma crescente as séries dos resíduos estimados, i.e. $\hat{\mu}_{1}^{c}<\hat{\mu}_{2}^{c}<\ldots<\hat{\mu}_{T}^{c}$, onde $T$ é o número de observações. Os 15 por cento maiores e menores dos valores de $\left\{\hat{\mu}_{i}^{c}\right\}$ seriam, então, descartados. Cada um dos 70 por cento dos valores restantes passaria a ser considerado um possível atrator. Para cada um destes atratores seria estimada uma equação, como as (16) e (17). O $\tau$ estimado que apresentasse a menor soma dos quadrados dos resíduos seria o apropriado. Procedimento análogo poderia ser utilizado para o modelo M-TAR. Estes modelos passam a ser, então, denominados TAR e M-TAR consistentes e as estatísticas de teste serão agora a $t_{\max }^{c}$ e a $\phi^{c}$.

Se $\tau$ for desconhecido, a inferência dos valores de $\rho_{1}$ e $\rho_{2}$ juntamente com a imposição da restrição $\rho_{1}=\rho_{2}$ tornam-se problemáticas, porque aqui não há normalidade multivariada assintótica. Porém, Chan and Tong (1989) conjeturam que os modelos TAR e M-TAR consistentes apresentam normalidade 
assintótica nos coeficientes. Enders and Falk (1998) também acham que não ocorre o problema para amostras pequenas, desde que seja usada uma distribuição bootstrap para as estatísticas de máxima verossimilhança.

\subsection{Bolhas intrínsecas}

Froot and Obstfeld (1991) observaram que, quando uma bolha não se correlaciona com os fundamentos, ela cresce exogenamente a uma taxa esperada $1+r$ por período, já que não ocorre arbitragem. Portanto, a bolha e os fundamentos divergem a uma taxa explosiva. Porém, se correlacionada com os fundamentos, a bolha pode surgir mesmo sem a presença de fatores externos. Esta "bolha intrínseca" será função não linear dos dividendos correntes e representa uma reação excessiva do preço da ação em relação às variações dos dividendos. Partindo da condição de que o preço corrente da ação se igualaria ao valor presente descontado dos pagamentos de dividendos, mais o preço da ação do período seguinte (todos em termos reais), i.e.

$$
P_{t}=\mathrm{e}^{-r} E_{t}\left(D_{t}+P_{t+1}\right)
$$

a bolha racional seria dada por

$$
B_{t}=\mathrm{e}^{-r} E_{t}\left(B_{t+1}\right)
$$

do mesmo modo que em (7). Note que a equação 19 é uma variante de (5), com a taxa de desconto $r$ constante, o preço $P_{t}$ sendo o do início do período, e o dividendo $D_{t}$ sendo aquele pago ao final do período. $\mathrm{O} \log$ dos dividendos seguiria um caminho aleatório com drift $\mu$, i.e.

$$
d_{t+1}=\mu+d_{t}+\xi_{t+1}
$$

onde $\xi_{t+1} \sim N\left(0, \sigma^{2}\right)$. A bolha intrínseca, que é função não linear dos dividendos, satisfaria a equação (20):

$$
B\left(D_{t}\right)=c D_{t}^{\lambda}
$$

Aqui, o parâmetro $\lambda$ é a raiz positiva da equação quadrática

$$
\frac{\sigma^{2}}{2} \lambda^{2}+\mu \lambda-r=0
$$

onde $B\left(D_{t}\right)$ representa a bolha, e $c$ é uma constante arbitrária. O preço compatível com o modelo de valor presente $P_{t}^{f}$ seria proporcional aos dividendos:

$$
P_{t}^{f}=\kappa D_{t}
$$

onde

$$
\kappa=\left(e^{r}-e^{\left(\mu+(1 / 2) \sigma^{2}\right)}\right)^{-1}
$$

Aqui, $\mu$ e $\sigma^{2}$ representam a média e a variância estimadas a partir da equação 21 . Como o preço da ação incorpora tanto o compatível com o valor presente como o componente relacionado à bolha, temos que

$$
P_{t}=\kappa D_{t}+c D_{t}^{\lambda}
$$

Se o preço fundamental da ação implicar uma razão preço-dividendo $\kappa$ constante, a bolha torna-se função apenas dos dividendos correntes, e o preço poderá reagir excessivamente em relação a eles. Se apenas a constante $\kappa$ for significativa, não haverá bolha. Se verificada uma relação não linear entre o preço e os dividendos, a existência de bolha intrínseca não poderá ser descartada.

Para testar bolhas intrínsecas, Froot and Obstfeld (1991) estimaram os parâmetros de uma versão estocástica da equação não linear (26), i.e.

$$
P_{t}=\kappa D_{t}+c D_{t}^{\lambda}+\mu_{t}
$$


Como $D_{t}$ e $D_{t}^{\lambda}$ são as variáveis explicativas, os dados podem ser colineares para $\lambda \rightarrow 1$. Por isso, foi necessário restringir um dos coeficientes para estimar (27). Assim, $\lambda$ e $\kappa$ foram calculados de acordo com (23) e (25). Depois eles testaram o resíduo da equação de longo prazo utilizando os modelos convencionais de raiz unitária. Sendo rejeitada a hipótese de que o resíduo ajustado $\mu_{t}$ é $I(1)$, então $P_{t}, D_{t}$ e $D_{t}^{\lambda}$ devem ser não linearmente cointegrados, o que indicaria a presença de bolhas intrínsecas no longo prazo.

Froot and Obstfeld (1991) também verificaram se o preço da ação é função linear dos dividendos e também se a razão preço-dividendo $\kappa$ é constante, como na equação 26. Aqui, a hipótese nula foi ausência de bolha intrínseca. Eles rodaram a regressão da razão preço-dividendo contra uma constante e os dividendos. Se apenas a constante fosse significativa, haveria ausência de bolha. Se fosse verificada a relação não linear entre o preço e os dividendos $(c \neq 0)$, isto seria sinal de bolha intrínseca. Para $c$ estatisticamente positivo, isto significaria que, com o aumento dos dividendos, o preço da ação se tornaria cada vez mais sobrevalorizado em relação a seu valor fundamental. Com a redução dos dividendos, o componente relacionado à bolha seria reduzido e o preço se aproximaria do valor fundamental.

Uma forma alternativa e mais direta de se testar o modelo é verificar a cointegração entre uma transformação não linear das séries dos preços das ações e os dividendos (Ma and Kanas, 2004). Esta transformação para os preços das ações é dada por

$$
P_{t}^{*}=P_{t}-\bar{\kappa} D_{t}
$$

onde $\bar{\kappa}$ é a estimativa no ponto de $\kappa$, e $P_{t}^{*}$ é a série transformada dos preços das ações. A transformação para os dividendos é dada por

$$
D_{t}^{*}=D_{t}^{\bar{\lambda}}
$$

onde $\bar{\lambda}$ é a estimativa no ponto de $\lambda$, e $D_{t}^{*}$ é a série transformada dos dividendos. A relação de cointegração entre as séries transformadas é

$$
P_{t}^{*}=c D_{t}^{*}+\varepsilon_{t}
$$

A existência de cointegração entre $P_{t}^{*}$ e $D_{t}^{*}$ significa que $P_{t}, D_{t}$ e $D_{t}^{\lambda}$ também são cointegrados, havendo sinal de bolha intrínseca. Este procedimento também pode ser considerado uma cointegração não linear (Granger and Hallman, 1991).

\section{ANÁLISE DOS RESULTADOS}

As séries mensais dos índices Bovespa e dos dividendos foram obtidas de Datastream, na seção Emerging Markets Data Base fornecida pela Standard \& Poors. Ambas as séries foram deflacionadas pelo Índice de Preços ao Consumidor Ampliado. O período analisado foi de julho de 1994 a dezembro de 2006. Tomamos o log natural para suavizar as séries, preservando o seu conteúdo informativo. Na Figura 1 estão as séries dos preços das ações e dos dividendos, assim como suas médias e desvios-padrões.

A ordem de integração das duas séries foi verificada através dos testes de raiz unitária Dickey and Fuller (1979), Dickey-Fuller aumentado (ADF) (Engle and Granger, 1987) e Phillips-Perron (PP) (1988) (Tabela 1). Estimamos, primeiramente, o modelo irrestrito incluindo constante e tendência no processo auto-regressivo. A ordem das defasagens foi escolhida utilizando os critérios de informação Akaike e Schwarz com nível de significância de 5 por cento. Encontramos que as séries são não estacionárias em níveis, mas tornam-se estacionárias em primeiras diferenças. Portanto, os preços das ações e os rendimentos dos dividendos em termos reais são integrados de primeira ordem (i.e. $I(1)$ ).

Na Tabela 2 apresentamos os resultados dos modelos de cointegração convencional com ajustamento simétrico (Johansen e Engle-Granger) e de cointegração threshold com ajustamento assimétrico (TAR, M-TAR, TAR consistente e M-TAR consistente). A rejeição da hipótese nula de não cointegração nos testes Johansen e Engle-Granger indica a presença de bolhas explosivas no mercado de ações brasileiro 
Figure 1: Índice Bovespa: Preços das ações $(P)$ e dividendos $(D)$, 1994-2006

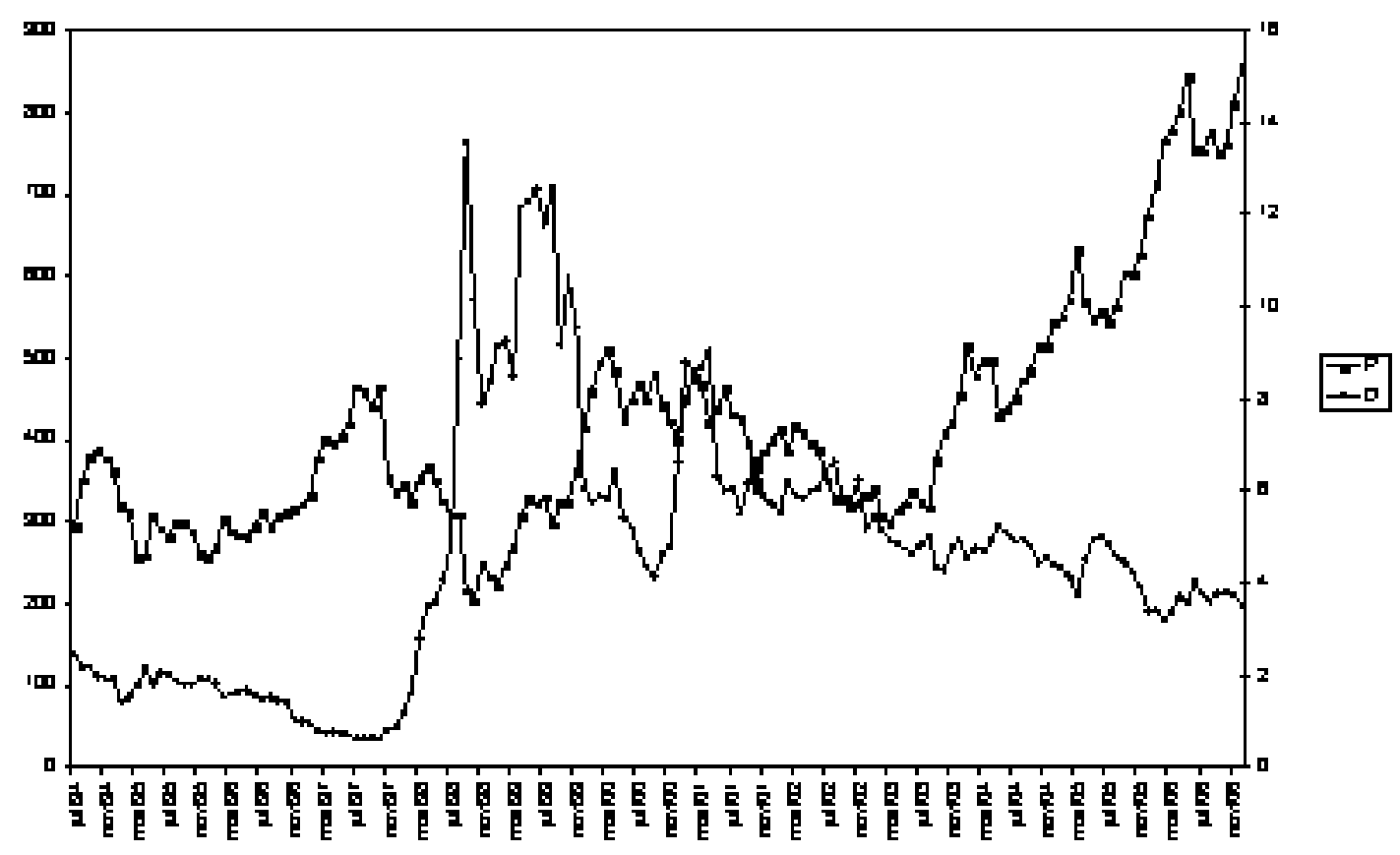

Table 1: Teste de raiz unitária para os preços das ações e os dividendos

\begin{tabular}{|l|c|c|c|c|c|c|c|c|}
\hline Série & \multicolumn{4}{|c|}{ Níveis } & \multicolumn{4}{c|}{ Primeiras diferenças } \\
\hline & $\mathrm{ADF}(\mathrm{l})$ & $\tau_{\text {crit }}$ & $\mathrm{PP}$ & $\tau_{\text {crit }}$ & $\mathrm{ADF}(\mathrm{l})$ & $\tau_{\text {crit }}$ & $\mathrm{PP}$ & $\tau_{\text {crit }}$ \\
\hline Preços & $-1.98^{* *}$ & -3.44 & -2.14 & -3.44 & -11.00 & -1.94 & -11.00 & -1.94 \\
Dividendos & $-1.62^{*}$ & -2.88 & $-1.67^{*}$ & -2.88 & -9.39 & -1.94 & -9.54 & -1.94 \\
\hline
\end{tabular}

: Notas: $A D F(\Delta)$ é o teste Dickey-Fuller aumentado com o número ótimo de defasagens entre parênteses (i.e. 1); (critério Akaike-Schwarz) PP é o teste Phillips-Perron $\tau_{c r i t}$ representa os valores críticos ao nível de significância de $5 \%$; ${ }^{*}$ teste com constante; ${ }^{* *}$ teste com constante e tendência. 
e rejeição do modelo de valor presente no curto prazo. Mas também há evidência de bolhas que estouram periodicamente nos modelos TAR e M-TAR consistentes. De fato, os valores da estatística $F$ para rejeição da hipótese nula de cointegração $\left(\phi^{c}\right)$ para os modelos TAR e M-TAR consistente foram de 6.88 e 7.96, respectivamente, o que significa que somente em alguns períodos ocorreram desvios da relação de longo prazo entre preços das ações e dividendos, não sendo possível rejeitar o modelo de valor presente no longo prazo.

Table 2: Estimativa de bolha nos preços dos ativos

\begin{tabular}{|l|c|c|c|c|c|c|}
\hline & Johansen & Engle-Granger & TAR & M-TAR & $T A R_{\text {Consistente }}$ & $M-T A R_{\text {Consistente }}$ \\
\hline$\lambda_{\text {traço }}$ & $6.89(\mathrm{C}, 2)$ & - & - & - & - & - \\
$\rho_{1}$ & - & $-0.022(1)$ & -0.018 & -0.068 & $-0.026(2)$ & $-0.016(1)$ \\
estatística $t$ & & $(-1.51)$ & $(-1.15)$ & $(-0.05)$ & $(-1.65)$ & $(-0.88)$ \\
$\rho_{2}$ & - & - & -0.142 & -0.359 & -0.166 & -0.450 \\
estatística $t$ & & & $(-2.11)$ & $(-3.48)$ & $(-3.42)$ & $(-0.00)$ \\
AIC & - & 546.84 & 532.51 & 545.87 & 520.43 & 540.33 \\
BIC & - & 552.83 & 550.37 & 551.87 & 544.23 & 549.30 \\
$\tau$ & - & - & - & - & 0.663 & -0.437 \\
$\phi, \phi^{c}$ & - & - & 4.64 & 5.22 & 6.88 & 7.96 \\
$\rho_{1}=\rho_{2}$ & - & - & 5.97 & 3.27 & 7.98 & 7.12 \\
valor $p$ & & & $(0.016)$ & $(0.072)$ & $(0.005)$ & $(0.008)$ \\
\hline
\end{tabular}

\footnotetext{
: Notas: Número de observações: 152 ; Valores críticos: $\lambda_{\text {traço(1\%) }}=19.96, \tau_{(10 \%)}=-3.03, \phi_{\operatorname{TAR}(10 \%)}=4.92, \phi_{\mathrm{M}-\mathrm{TAR}(10 \%)}=5.45$, $\phi_{\mathrm{TAR}(10 \%)}^{c}=6.02, \phi_{\mathrm{M}-\mathrm{TAR}(5 \%)}^{c}=6.86 ; \rho_{1}$ e $\rho_{2}$ são os coeficientes dos resíduos defasados $\left(\hat{\mu}_{t-1}\right)$; Os valores entre parênteses são para as primeiras diferenças dos resíduos defasados de $\triangle \hat{\mu}_{t-i}$ e do componente determinístico.
}

Os resultados dos critérios de informação Akaike (AIC) e Schwarz (BIC) sugeriram que o M-TAR consistente é o melhor modelo e que os desvios do caminho de equilíbrio de longo prazo foram revertidos rapidamente, como seria de se esperar de um modelo M-TAR (Enders and Granger (1998), Enders and Siklos (2001)). Também foi confirmada a conjectura de Chan (1993) de que o valor do threshold ( $\tau$ ) não necessariamente coincide com o atrator zero em dados reais. De fato, para valores situados entre 0 threshold e zero, houve persistência e não ocorreu reversão à média. Porém, para valores que ultrapassaram o valor do threshold houve rápida reversão. Dentro dessas bandas, as discrepâncias foram insignificantes, de modo que não podem ser consideradas como desvios dos fundamentos. Os valores dos thresholds nos modelos TAR e M-TAR consistentes foram de 0.663 e -0.437 , respectivamente (Figuras 2 e 3 ).

As condições necessárias e suficientes para a estacionaridade de $\{\hat{\mu}\}$ de $\rho_{1}, \rho_{2}<0$ e $\left(1+\rho_{1}\right)(1+$ $\left.\rho_{2}\right)<1$ foram verificadas. Os coeficientes dos resíduos defasados $\hat{\mu}_{t-1}\left(\rho_{1}\right.$ e $\left.\rho_{2}\right)$ foram de -0.166 e -0.026 para o modelo TAR consistente e -0.450 e -0.016 para o M-TAR consistente, respectivamente. A hipótese nula de ajustamento simétrico $\left(\rho_{1}=\rho_{2}\right)$ foi rejeitada ao nível de significância de um por cento. Os valores da estatística $F$ foram de 7.98 (TAR consistente) e 7.12 (M-TAR consistente). Além disso, os ajustamentos acima da equação de equilíbrio de longo prazo foram mais persistentes do que os desvios abaixo desse equilíbrio $\left(\left|\rho_{2}\right|<\left|\rho_{1}\right|\right)$. Este resultado dá suporte à hipótese de que houve aumentos dos preços das ações no curto prazo (em relação aos preços fundamentais) que foram seguidos de crash. Porém, este resultado também significa que, no longo prazo, os preços se alinharam aos dividendos, representando corretamente os fundamentos.

Para testar a hipótese de bolhas intrínsecas, os coeficientes $\lambda$ e $\kappa$ foram estimados através dos parâmetros $\mu$ e $\sigma^{2}$ da equação 24, sendo $\lambda$ a raiz positiva da equação 22. A taxa de desconto $r$ foi calculada por $R=\exp (r)=\frac{P_{t+1}+D_{t+1}}{P}$, sendo $\bar{r}$ a média aritmética de $\ln R$. 
Figure 2: Modelo TAR: threshold estimado

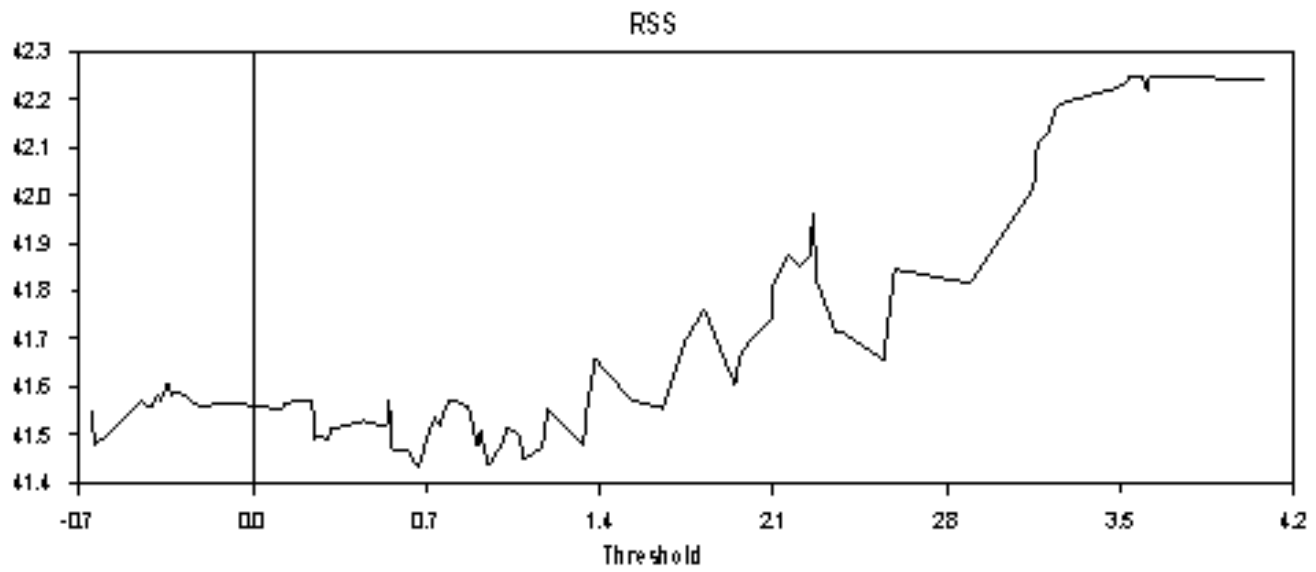

Figure 3: Modelo M-TAR: threshold estimado

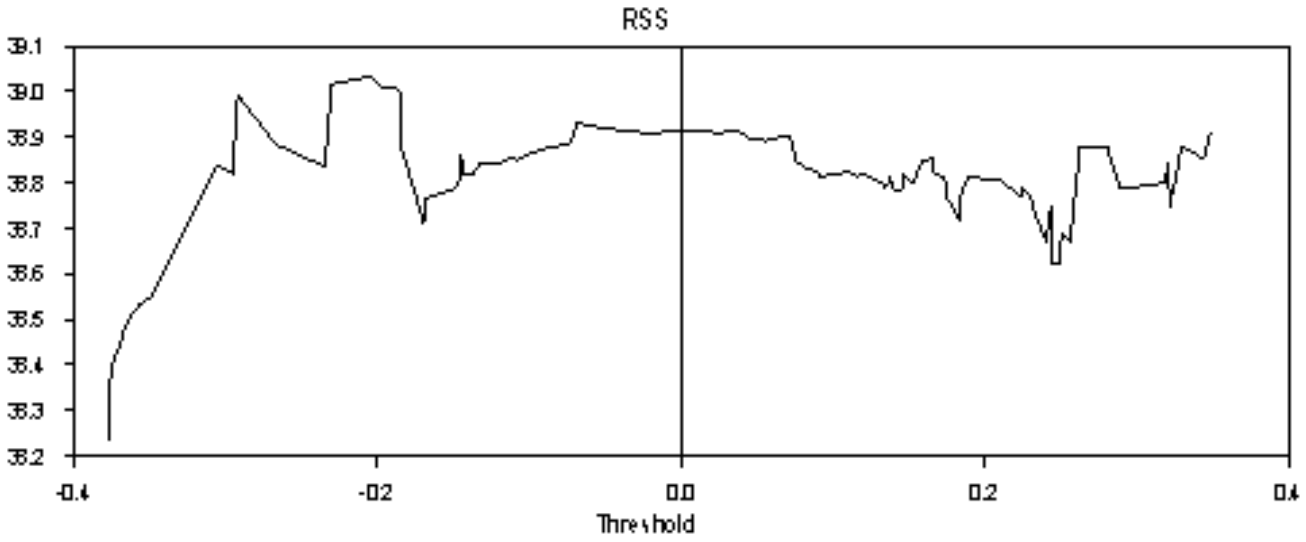


Assim como em Froot e Obstfeld, ao derivar a equação 26 e, conseqüentemente, a equação 27, supusemos que os logs dos dividendos seguem um martingale geométrico com drift, que os resíduos são i.i.d. e que a taxa de desconto é constante. Isto pressupõe testar a especificação da equação 21 e do resíduo, além da estacionaridade da taxa de desconto. Por isso, os parâmetros da equação dos dividendos foram consistentemente estimados via Newey-West e a verificação de se os resíduos são de fato i.i.d. pode ser feita através do teste BDS (Brock et al., 1996). A estacionaridade da taxa de desconto foi verificada pelos testes ADF e Phillips-Perron. A desigualdade $r>\mu+\frac{\sigma^{2}}{2}$ implicou $\lambda>1$. Foi esta não linearidade explosiva que fez com que a expectativa da bolha $B\left(D_{t}\right)$ crescesse à taxa $r$.

Os resultados dos testes de bolhas intrínsecas estão na Tabela 3. Estimamos tanto um modelo restrito como um irrestrito usando o modelo de dois passos de Engle and Granger (1987), i.e. $P_{t}=$ $\kappa D_{t}+c_{1} D_{t}^{\lambda}+\mu_{t}$ com $\triangle \hat{\mu}_{t}=\rho_{1} \hat{\mu}_{t-1}+\sum_{i=1}^{l} \gamma_{i} \triangle \hat{\mu}_{t-1}+\varepsilon_{t}$, onde $\rho_{1}$ é o coeficiente do resíduo defasado $\mu_{t-1}$. O modelo restrito foi estimado usando a restrição dos coeficientes $C_{0}=\kappa$ e $\lambda=\bar{\lambda}$. No modelo irrestrito, $\lambda$ foi estimado livremente. $O$ teste de Wald $F_{\text {teste }} \lambda=\bar{\lambda}$ foi utilizado para verificar restrição dos coeficientes.

Table 3: Estimativa de bolhas intrínsecas

\begin{tabular}{|l|c|c|c|c|c|c|}
\hline Modelo & $c_{1}$ & $\lambda_{\text {traço }}$ & $\lambda$ & $\rho_{1}$ & $F_{\text {teste }} \lambda=\bar{\lambda}$ & $D W$ \\
\hline \multirow{2}{*}{ Restrito } & $1435.5^{* * *}$ & - & - & $-0.005(C)$ & - & 1.93 \\
\cline { 2 - 7 } & $(55.45)$ & & & $(-1.82)$ & & \\
\hline \multirow{2}{*}{ Irrestrito } & $1471.3^{* * *}$ & - & $1.057^{* * *}$ & -0.068 & 3.222 & 2.03 \\
\cline { 2 - 7 } & $(67.08)$ & & $(360.6)$ & $(-1.634)$ & {$[0.0747]$} & \\
\hline Alternativo & - & 7.27 & - & - & - & - \\
\hline
\end{tabular}

: Notas: Número de observações: $152 ; \rho_{1}$ representa os coeficientes dos resíduos defasados $\varepsilon_{t-1}$; $C$ e $T$ representam a constante e a tendência na equação dos resíduos da equação de cointegração; * , **, *** representam significância estatística a $10 \%, 5 \%$ e $1 \%$, respectivamente; $D W$ é a estatística Durbin-Watson para a regressão dos resíduos; $\tau_{\text {crit rest }(10 \%)}=2.57$ e $\tau_{\text {crit irrest }(10 \%)}=-1.615$ são os valores críticos para o teste de raiz unitária Phillips-Perron; Valor crítico da estatística traço: $\tau_{\text {traço }(5 \%)}=20.26$.

Os dois modelos foram estimados por MQO não lineares e os valores dos coeficientes do modelo restrito calculados por (23) e (25) foram $\lambda=1.032$ e $\kappa=1592.9$. Os valores da média e do erro-padrão utilizados nos cálculos dos coeficientes da equação 25 foram $\mu=0.01848$ e $\sigma=0.0202$, e a taxa de desconto foi $\bar{r}=0.0193$. Os testes Phillips-Perron e ADF rejeitaram a hipótese nula de raiz unitária para $\ln r$. A estatística do teste BDF para os resíduos da equação 20 foi de 0.0006 com probabilidade 0.178 (bootstrap).

Também consideramos um modelo alternativo, onde utilizamos o modelo de cointegração de Johansen para as séries transformadas dos preços das ações e dos dividendos (equação 30).

Os coeficientes do termo relacionado à bolha $\left(c_{1}\right)$ das regressões dos modelos restrito e irrestrito foram estatisticamente significativos (a um por cento), o que sugere que, com o aumento dos dividendos, os preços das ações se tornaram crescentemente sobrevalorizados em relação ao valor fundamental. A estimativa do coeficiente $\lambda$ (de 1.057) não foi estatisticamente diferente do seu valor calculado $\lambda=\bar{\lambda}=1.032$. Além disso, como $\lambda>1$, a desigualdade $r>\mu+\frac{\sigma^{2}}{2}$ foi satisfeita, o que implica crescimento não linear explosivo da expectativa de bolha $B\left(D_{t}\right)$ à taxa $r$, de acordo com a equação 20 .

Porém, os coeficientes dos resíduos defasados $\left(\rho_{1}\right)$ das equações de cointegração dos modelos restrito e irrestrito (iguais a -0.005 e -0.068 , respectivamente) foram estatisticamente não significativos a 10 por cento. Assim, a hipótese de que os resíduos ajustados $\hat{\varepsilon}_{t}$ são $I(1)$ não pode ser rejeitada, o que sugere que $P_{t}, D_{t}$ e $D_{t}^{\lambda}$ não são cointegrados. O coeficiente de 7.27 do modelo alternativo confirmou esses resultados. Portanto, se houve bolha nos preços das ações listadas no índice Bovespa, a 
mesma deve ter sido causada por eventos extrínsecos, e não pela relação não linear dos preços com os dividendos. As notas da Tabela 3 fornecem mais detalhes sobre este teste.

\section{CONCLUSÃO}

Neste trabalho investigamos a presença de bolhas racionais no índice Bovespa. Nossos resultados indicam a presença de bolhas explosivas e de bolhas que estouram periodicamente. Estas últimas foram detectadas por modelos TAR e M-TAR consistentes. Como o melhor modelo foi o M-TAR consistente, isto significa que o modelo de valor presente deve ser válido apenas no longo prazo, e que o ajustamento do curto ao longo prazo foi assimétrico. Os ajustamentos acima da equação de equilíbrio de longo prazo foram mais persistentes do que os desvios abaixo desse equilíbrio, o que não permite descartar a hipótese de aumentos dos preços das ações no curto prazo (em relação aos preços fundamentais) seguidos de crash. Estas bolhas racionais foram provavelmente causadas por fatores extrínsecos. De fato, não houve cointegração não linear entre os preços das ações e os dividendos, o que sugere a ausência de bolhas intrínsecas.

\section{BIBLIOGRAPHY}

Bidarkota, P. \& Dupoyet, B. (2007). Intrinsic bubbles and fat tails in stock prices: A note. Macroeconomic Dynamics, 11:405-422.

Bikhchandani, S. \& Sharma, S. (2001). Herd behavior in financial markets: A review. IMF Staff Papers, 47:279-310.

Blanchard, O. (1979). Speculative bubbles, crashes and rational expectations. Economics Letters, 3:387389.

Blanchard, O. \& Watson, M. W. (1982). Bubbles, rational expectations and financial markets. Technical Report 945, NBER.

Bohl, M. T. (2003). Periodically collapsing bubbles in the US stock market? International Review of Economics and Finance, 12:385-397.

Bohl, M. T. \& Siklos, P. L. (2004). The present value model of US stock prices redux: A new testing strategy and some evidence. Quarterly Review of Economics and Finance, 44:208-223.

Brock, W. A., Dechert, W. D., Scheikman, J. A., \& LeBaron, B. (1996). A test for independence based on the correlation dimension. Econometric Reviews, 15:197-235.

Campbell, J. Y. \& Shiller, R. J. (1988). The dividend-price ratio and expectations of future dividends and discount factors. Review of Financial Studies, 1:195-228.

Caner, M. \& Hansen, B. E. (2001). Threshold autoregression with a unit root. Econometrica, 69:15551596.

Chan, K. S. (1993). Consistency and limiting distribution of the least squares estimator of a threshold autoregressive model. The Annals of Statistics, 21:520-533.

Chan, K. S. \& Tong, H. (1989). A survey of the statistical analysis of a univariate threshold autoregressive model. In Mariano, R., editor, Advances in Statistical Analysis and Statistical Computing: Theory and Applications, pages 1-42. JAI Press Inc., Greenwich, Conn. 
Diba, B. T. \& Grossman, H. I. (1987). On the interception of rational bubbles. Quarterly Journal of Economics, 102:697-700.

Diba, B. T. \& Grossman, H. I. (1988a). Explosive rational bubbles in stock prices? American Economic Review, 78:520-530.

Diba, B. T. \& Grossman, H. I. (1988b). The theory of rational bubbles in stock prices. Economic Journal, 98:746-754.

Dickey, D. A. \& Fuller, W. A. (1979). Distribution of the estimators for autoregressive time series with a unit root. Journal of the American Statistical Association, 74:427-431.

Driffill, J. \& Sola, M. (1998). Intrinsic bubbles and regime switching. Journal of Monetary Economics, 42:357-373.

Enders, W. \& Falk, B. (1998). Threshold-autoregressive, median-unbiased and cointegration tests of purchasing power parity. International Journal of Forecasting, 14:171-186.

Enders, W. \& Granger, W. J. (1998). Unit-root tests and asymmetric adjustment with an example using the term structure of interest rates. Journal of Business \& Economic Statistics, 16:304-311.

Enders, W. \& Siklos, P. (2001). Cointegration and threshold adjustment. Journal of Business \& Economic Statistics, 19:166-176.

Engle, R. \& Granger, C. L. W. (1987). Cointegration and error-correction: Representation, estimation and testing. Econometrica, 55:251-276.

Evans, G. (1991). Pitfalls in testing for explosive bubbles in asset prices. American Economic Review, 81:922-930.

Froot, K. \& Obstfeld, M. (1991). Intrinsic bubbles: The case of stock prices. American Economic Review, 81:1189-1214.

Gauthier, C. \& Li, F. C. (2004). Linking real activity and financial markets: The bonds, equity and money. Technical Report Working Paper 2006-42, Bank of Canada.

Granger, C. W. J. \& Hallman, J. (1991). Long memory processes with attractors. Oxford Bulletin of Economics and Statistics, 53:11-26.

Hall, S. G. \& Sola, M. (1993). Testing for collapsing bubbles: An endogenous switching ADF test. Technical Report Discussion Paper 15, London Business School.

Hamilton, J. D. \& Whiteman, C. H. (1985). The observable implications of self-fulfilling expectations. Journal of Monetary Economics, 16:353-373.

Helbling, T. \& Terrones, M. (2003). When bubbles burst. IMF World Economic Outlook, 61-94.

Herrera, S. \& Perry, G. E. (2003). Tropical bubbles: Asset prices in Latin America, 1980-2001. In Hunter, W. C., Kaufman, G. G., \& Pomerleano, M., editors, Asset Prices Bubbles: The Implications for Monetary, Regulatory and International Policies. MIT Press, Cambridge, MA.

Johansen, S. (1988). Statistical analysis of cointegration vectors. Journal of Economic Dynamics and Control, 12:231-254.

Johansen, S. (1991). Estimation and hypothesis testing of cointegration vectors in Gaussian vector autoregressive models. Econometrica, 59:1551-1580. 
Ma, Y. \& Kanas, A. (2004). Intrinsic bubbles revisited: Evidence from nonlinear cointegration and forecasting. Journal of Forecasting, 23:237-250.

Martin, D. L. M., Kayo, E. K., Kimura, H., \& Nakamura, W. T. (2004). Identificando bolhas especulativas racionais no IBOVESPA (pós-Plano Real) a partir de regimes markovianos de conversão. Economia, 5:219-252.

Mundial, B. (1997). Private Capital Flows to Developing Countries: The Road to Financial Integration. World Bank.

Nunes, M. S., Da Costa Jr, N., \& Meurer, R. (2005). A relação entre o mercado de ações e as variáveis macroeconômicas: Uma análise econométrica para o Brasil. Revista Brasileira de Economia, 59:586607.

Payne, J. E. \& Waters, G. A. (2005). Periodically collapsing negative bubbles? Applied Financial Economics Letters, 1:65-69.

Petruccelli, J. D. \& Woolford, S. W. (1984). A threshold AR(1) model. Journal of Applied Probability, 21:270286.

Santos, M. S. \& Woodford, M. (1997). Rational asset pricing bubbles. Econometrica, 65:19-58.

Sarno, L. \& Taylor, M. (2003). An empirical investigation of asset price bubbles in Latin American emerging financial markets. Applied Financial Economics, 13:635-643.

Sarno, L. \& Taylor, M. P. (1999a). Hot money, accounting labels and the permanence of capital flows to developing countries: An empirical investigation. Journal of Development Economics, 59:337-364.

Sarno, L. \& Taylor, M. P. (1999b). The persistence of capital inflows and the behavior of stock prices in East Asian emerging markets: Some empirical evidence. Journal of International Money and Finance, 18:637-657.

Taylor, M. \& Peel, D. (1998). Periodically collapsing stock price bubbles: A robust test. Economics Letters, 61:221-228.

Tong, H. (1983). Threshold Models in Non-Linear Time Series Analysis. Springer-Verlag, New York.

Van Norden, S. (1996). Regime switching as a test for exchange rate bubbles. Journal of Applied Econometrics, 11:219-251.

Van Norden, S. \& Vigfusson, R. (1998). Avoiding the pitfalls: Can regime-switching tests reliably detect bubbles? Studies in nonlinear dynamics and econometrics, 3:1-22. 